\title{
RENORMALIZATION GROUP FLOWS IN THE DEFORMED ADS/CFT CORRESPONDENCE
}

\author{
NICK EVANS \\ Department of Physics and Astronomy, University of Southampton, Highfield, \\ Southampton, SO17 1BJ, UK
}

\begin{abstract}
In the AdS/CFT correspondence motion in the radial direction of the AdS space is identified with renormalization group flow in the field theory. For the $\mathrm{N}=4$ YangMills theory this motion is trivial. More interesting examples of renormalization group flow occur when the $\mathrm{N}=4$ theory is deformed. Aspects of the flows are discussed for the $\mathrm{N}=4$ theory on its moduli space, and deformed to $\mathrm{N}=2$ in the infra-red within the context of 5d SUGRA. 10d lifts and brane probing are crucial tools for linking the spacetime backgrounds to the dual field theory.
\end{abstract}

\section{Introduction}

There is now a well established and remarkable correspondence between $\mathrm{N}=4$ super Yang-Mills (SYM) gauge theory in $3+1$ dimensions and type IIB string theory on $A d S_{5} \times S^{5}$ spacetimella. The renormalization group (RG) appears to be an integral component of the duality with changes in RG scale in the gauge theory corresponding to motion in the "holographic" radial direction of the $A d S_{5}$ space on the gravity side 3 . As will be reviewed below this role for $\mathrm{RG}$ flow was deduced in the original case of the $\mathrm{N}=4 \mathrm{SYM}$ theory. The RG flow in this theory is trivial however since the theory is conformal. There has therefore been considerable interest in studying more complicated examples of the correspondence where the RG flow is more dynamic. A sensible way in which to construct such theories is to begin with the $\mathrm{N}=4$ conjecture and use the symmetries on each side of the duality to understand how to include relevant deformations 1 . This article will attempt to provide a brief overview of the state of such investigations. To maximally simplify the effort on the string theory side of the duality initial studies were performed in the 5 dimensional supergravity (SUGRA) theory describing the string theory at long distances relative to the $S^{5} / A d S_{5}$ radius. As will emerge though the results have lead back to elements (such as branes) of the full string theory requiring more complex analysis. In addition the deformed SUGRA backgrounds discovered need considerable work to extract/confirm properties of their dual field theories. In this respect brane probing has been found to be a sharp tool. As examples of nonconformal theories I will discuss the $\mathrm{N}=4$ theory on moduli space as investigated in $\mathrm{G}$ and the $\mathrm{N}=4$ theory deformed by the inclusion of a mass term to $\mathrm{N}=2 \mathrm{~A}$. 1 . Much of my discussion is based on work with M. Petrini and C.V. Johnson.

\section{The AdS/CFT Correspondence}

The $A d S / \mathrm{CFT}$ correspondence is between $\mathrm{N}=4 \mathrm{SYM}$ in $3+1$ dimensions (a theory with four adjoint spinors, 6 real scalars and an SU(N) gauge group) and Type IIB string theory on the space $A d S_{5} \times S^{5}$. The $A d S$ space is given by the metric

$$
d s^{2}=\frac{d u^{2}}{u^{2}}+u^{2} d x^{\mu} d x_{\mu}
$$


and may therefore be thought of as a stack of $3+1$ dimensional Minkowski spaces with a warp factor in the perpendicular $u$ direction. As the correspondence was first proposed the N=4 SYM theory lived on the top most such Minkowski sheet at $u=\infty$. The bulk string theory at long distances may be replaced by Type IIB supergravity. The $\mathrm{N}=4 \mathrm{SYM}$ theory and the bulk states therefore naturally interact at the boundary. We allow all action terms of the form

$$
S=\int d x^{4} \mathcal{O}_{F T} \Phi_{0}
$$

where $\mathcal{O}_{F T}$ is a gauge invariant operator in the field theory and $\Phi_{0}$ is the boundary value of some supergravity field. Of course only terms that respect the global symmetries of each theory are allowed as we will discuss shortly. The vevs of supergravity fields at the boundary act as source terms in the field theory. It is immediately apparent how one would go about introducing conformal symmetry breaking operators into the boundary theory. Operators such as $\mathcal{O}_{F T} J$ can give rise to mass terms and operators of the form $\left|\mathcal{O}_{F T}-J\right|^{2}$ can be used to force the expectation values of fields to explore the moduli space of a theory.

The full statement of the duality is that the action of the classical (weakly coupled) supergravity theory evaluated with appropriate boundary conditions on the fields is the full generating functional for the quantum N=4 SYM theory (at large 'tHooft coupling $g^{2} N$ ) with the corresponding source terms.

The most immediate check is that the global symmetries of the two theories match. The $\mathrm{N}=4 \mathrm{SYM}$ theory has a $\mathrm{SU}(4)_{R}$ global symmetry. This is matched by the isometry group of the $S^{5}$ on the supergravity side of the duality. The $\mathrm{N}=4$ SYM is also conformal thus having a super-conformal symmetry group $\mathrm{SO}(2,4)$ for example there are dilatations of the form

$$
x \rightarrow e^{\alpha} x, \quad \phi \rightarrow e^{-\alpha} \phi
$$

that leave the massless scalar action invariant

$$
\int d x^{4} \partial^{\mu} \phi \partial_{\mu} \phi
$$

The supergravity theory is not conformal though (there are many mass scales associated with different Kaluza Klein states on the $S^{5}$ ) so this symmetry must be realized as a pure spacetime symmetry. The transformation on $x$ in (3) can be made a symmetry of the line element in (1) provided

$$
u \rightarrow e^{-\alpha} u
$$

The full $\mathrm{SO}(2,4)$ symmetry can also be realized as a spacetime symmetry.

We have just seen that the duality implies that the radial $u$ direction of $A d S$ transforms as a mass dimension under dilatations in the field theory. The natural correspondence to make for motion in $u$ is therefore change of RG scale in the field theory! We now let the field theory live on any of the Minkowski slices of $A d S_{5}$ but the action defined there by the supergravity field vevs is that of the field theory at a different RG scale. For the gravity dual to the $\mathrm{N}=4 \mathrm{SYM}$ at the origin of moduli space there are no sources switched on and hence no supergravity field vevs. The supergravity theory looks the same on every Minkowski space slice through $A d S_{5}$.

rome00: submitted to World Scientific on November 16, 2018 
This, of course, is a result of the $\mathrm{N}=4$ theory being conformal. Let us now think about some examples of related theories where the conformal symmetry is broken by the introduction of relevant operators.

\section{$3 \quad \mathrm{~N}=4$ SYM on Moduli Space}

The moduli space of the N=4 SYM theory is characterized by a vev for the gauge invariant quantity $\operatorname{tr} \phi^{2}$ so we must include a source term $\left|\operatorname{tr} \phi^{2}-J\right|^{2}$. There are six real scalar fields transforming as the 6 of $\mathrm{SU}(4)_{R}$ so $\operatorname{tr} \phi^{2}$ is in general a $6 \mathrm{x} 6$ matrix. The symmetric piece is the 20 representation of $\mathrm{SU}(4)_{R}$ so we can look for a source in the 20. The simplist supergravity analysis is to use the 5 dimensional truncation of Type IIB supergravity on $\mathrm{AdS}_{5} \times S^{5}$ which is valid on scales much larger than the radius of curvature and consists of only the lightest Kaluza Klein states. One of these is a scalar field in the 20 representation, $\alpha$. Even the truncated theory is quite complicated with the scalar fields transforming under the caset space $E_{6} / U S p(8)$. An expression for the potential was though calculated in 9 in the 1980s and we can proceed with those results. A notable feature of the potentials is that they are unbounded from below! In fact this is not as pathological as it first seems since the energy of the scalars receives a contribution from the positive curvature of the spacetime. This contribution allows a limited negative curvature.

As a sample flow consider turning on a vev $\operatorname{tr} \phi^{2}=\operatorname{diag}(1,1,1,1,-2,-2)$ 目 which corresponds to considering a particular element of the 20 . We can look for non-zero solutions of the equation of motion of the appropriate scalar in the supergravity. The scalar vev in the supergravity setting though can give rise to a backreaction on the metric. We will only consider variation in the $u$ direction so we can parametrize the metric as (writing $u=e^{y}$ )

$$
d s^{2}=d y^{2}+e^{2 A(y)} d x^{\mu} d x_{\mu}
$$

The equations of motion for the scalar $\rho=e^{\alpha}$ and $A$ are

$$
\frac{\partial \rho}{\partial y}=-\frac{2}{\sqrt{6}}\left(\rho^{5}-\frac{1}{\rho}\right), \quad \frac{\partial A}{\partial y}=\frac{1}{3}\left(\rho^{4}+\frac{1}{2 \rho^{2}}\right)
$$

In Fig 1 some sample solutions are shown. For large $y$ (the UV) $\phi \sim y$, the $A d S$ limit, and $\rho \sim \exp (2 y)$, showing that the deformation indeed has dimension 2. For small y $\rho$ grows (as the scalar vev rolls down the unbounded potential) in a way reminiscent of the RG flow of a dimension 2 operator in the field theory. A worrying aspect of the solutions is that the spacetime becomes singular at some finite value of $y$. Are these spacetimes good solutions? The singularities are an indication that the SUGRA theory is not the full story. The AdS/CFT duality is with the $\alpha^{\prime} \rightarrow 0$ limit of the full closed string theory and in particular we can expect to find D-brane configurations occuring. The SUGRA theory does not know about D-branes so will become singular where its knowledge fails. Remember that the correspondence was deduced by constructing the $\mathrm{N}=4$ gauge theory as the low energy description of open strings living on the surface of a coincident stack of D3 branes at $y=-\infty$. To place the theory on its moduli space we would separate the D-branes. In $\mathbf{O}_{\text {it }}$ was shown that this metric is indeed that describing the SUGRA background about such a D-brane distribution. These are therefore physical solutions.

rome00: submitted to World Scientific on November 16, 2018 

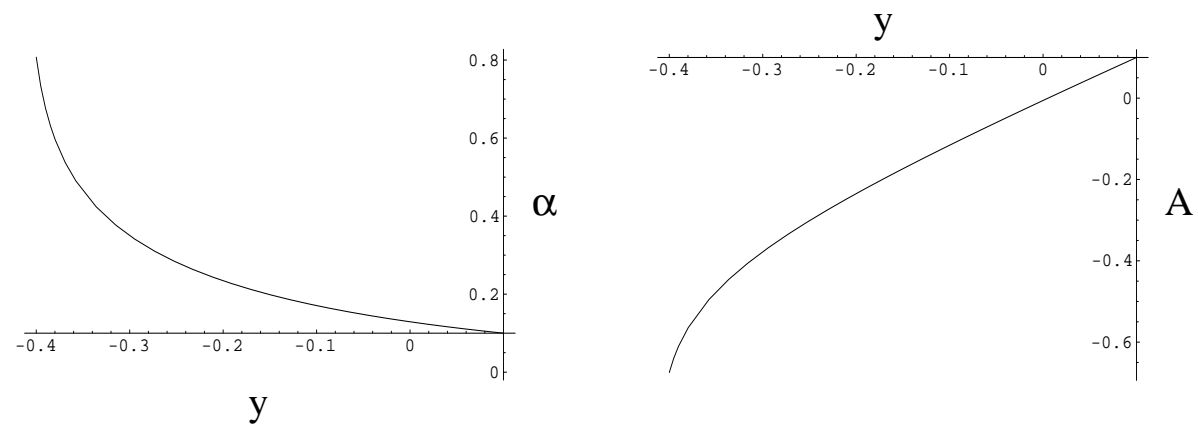

Figure 1. Sample solutions of the 5 d SUGRA scalars for the $\mathrm{N}=4$ theory on moduli space.

So far we have not shown any hard evidence for the claimed duality between these spacetimes and the $\mathrm{N}=4$ theory on moduli space. To do so we need a concrete tool that makes a connection between the two descriptions. Such a tool is brane probing; we imagine separating one of the D3 branes from the continuous distribution and move it about in the spacetime. The low energy description of the D3 brane is given by the Born-Infeld action and describes the U(1) gauge theory from the open string spectrum on its surface. The important point is that the scalar fields in the field theory on the brane correspond to the position of the brane in the spacetime. The action is

$$
S=-\mu \int d^{4} \xi \operatorname{det}^{1 / 2}\left[G_{a b}+e^{-\phi / 2} F_{a b}\right]+\mu \int C^{4}
$$

where $\mu$ is the D3 tension, $\phi$ the dilaton, $F^{a b}$ the gauge potential on the brane, $C^{4}$ the Ramond Ramond 4-form potential to which a D3 couples and finally $G^{a b}$ is the pull back of the spacetime metric

$$
G^{a b}=G_{\mu \nu} \frac{\partial x^{\mu}}{\partial \xi^{a}} \frac{\partial x^{\nu}}{\partial \xi^{b}}
$$

The probe therefore provides the link between the background spacetime metric and the low energy field theory variables.

Of course to perform the probe calculation we need the full 10D SUGRA solution rather than just the $5 \mathrm{D}$ solution. The use of the $5 \mathrm{D}$ truncation though is that for any solution there is automatically a lift to $10 \mathrm{D}$ though one requires considerable technology to perform the lift. Thankfully Pilch and Warnerd are experts in such matters and have provided us with the lift:

$$
d s^{2}=\frac{X^{1 / 2}}{\rho}\left(e^{2 A} d x^{2}+d r^{2}\right)+\frac{X^{1 / 2}}{\rho^{3}}\left(d \theta^{2}+\frac{\rho^{6} \cos ^{2} \theta}{X} d \Omega_{3}^{2}+\frac{\sin ^{2} \theta}{X} d \phi^{2}\right)
$$

where

$$
X=\cos ^{2} \theta+\rho^{6} \sin ^{2} \theta
$$

In addition the solution has a non-trivial 4 -from potential

$$
C^{4}=\frac{e^{4 A} X}{\rho^{2}} d x^{0} \wedge d x^{1} \wedge d x^{2} \wedge d x^{3} \wedge
$$


We may now perform the brane probe by substituting this background spacetime into the Born Infeld action. For a static probe we find the action vanishes exactly no matter the position of the brane in the 6 transverse dimensions. In the U(1) field theory on the brane's surface we have found a $6 \mathrm{~d}$ moduli space for the scalar field as expected in the $\mathrm{N}=4$ theory. Further allowing small velocities in these directions, after a shift in coordinate definitions in the $r-\theta$ plane, we find

$$
L=\frac{1}{2} \mu\left(\dot{r}^{2}+r^{2} d \Omega_{5}^{2}\right)
$$

which is a canonical kinetic term. This is to be compared to the $\mathrm{N}=4$ kinetic term $\tau \Phi^{\dagger} \Phi$ from which we can see that the coupling is independent of $r$ or scalar vev. These two results match with our expectations for the $\mathrm{N}=4$ theory and provide evidence for the correspondence.

\section{$4 \mathrm{~N}=4$ Broken to $\mathrm{N}=2$}

A more interesting theory to study is one where we include amass term in the $\mathrm{N}=4 \mathrm{SYM}$ theory that breaks the symmetry to $\mathrm{N}=2$ in the IR 6 . should have a running coupling below the mass scale. We must therefore allow an appropriate scalar to become non-zero in the SUGRA, $m$. In addition one expects the $\mathrm{N}=2$ theory to have a moduli space parametrized by the vev $\operatorname{tr} \phi_{3}^{2}$ of the remaining massless, complex scalar. In the SUGRA this vev corresponds to the scalar $\rho$ discussed above in the $\mathrm{N}=4$ theory. There are again solutions of the SUGRA equations of motion where at large $y$ the scalar fields have vevs approaching zero as $\exp (-d y)$ where $\mathrm{d}$ is the dimension of the source. In the IR these vevs grow. The equations of motion allow solutions for any initial conditions so we must understand how these correspond to physics in the dual field theory. It is natural to fix the mass at some UV RG scale (value of y) and then look at solutions with varying $\rho$ (scalar vev) at that same scale so the flows can be parametrized by $\rho\left(y_{U V}\right)$. As pointed out in 6 the flows divide into two classes, those that fall down an unbounded potential and those that eventually meet a rising potential. Inbetween there is a critical "ridge" flow. Based on the analysis of such solutions at high temperature Gubser has proposed that the flows that meet the rising potential are unphysical. The remaining flows he identifies with different points on moduli space with the critical flow perhaps representing some special point on the $\mathrm{N}=2$ theory's moduli space. Let us now look for confirmations of these identifications.

The $\mathrm{N}=2$ field theory has been studied in 11 . There are indeed singular points on the moduli space where monopoles become massless. When the theory is broken to $\mathrm{N}=1$ by the inclusion of an extra mass $M$ for the massless adjoint matter superfield of the $\mathrm{N}=2$ theory a potential develops that pins the vacua of the $\mathrm{N}=1$ theory to these singular points. The mass $M$ can be introduced as a third scalar in the SUGRAl although now only numerical solutions of the equations of motion are possible. Again sensible boundary conditions are to fix $m$ and $M \ll m$ at some $y_{U V}$ and the solutions are then parametrized by $\rho\left(y_{U V}\right)$. In Fig 2 the value of the potential at varying y values is shown for each solution. Solutions to the right of the ridge flow still meet a rising potential as they do in the $\mathrm{N}=2$ solution. Now though solutions to the left also begin to meet rising potentials in the IR. Eventually only

rome00: submitted to World Scientific on November 16, 2018 

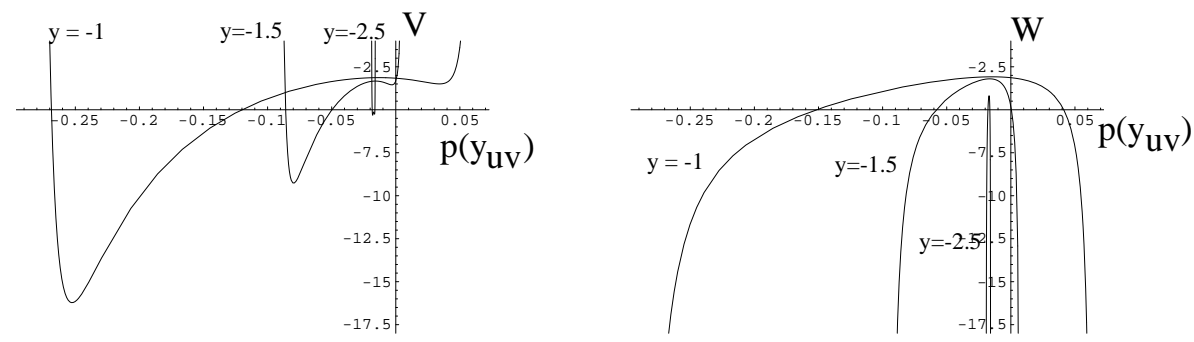

Figure 2. The potential and superpotential seen by $5 \mathrm{~d}$ SUGRA flows in the $\mathrm{N}=4$ broken to $\mathrm{N}=2$ and then sequentially to $\mathrm{N}=1$ theory, parametrized by $\rho\left(y_{U V}\right)$, as a function of radial coordinate.

the ridge flow itself is still crossing a falling potential. By Gubser's criteria only this single ridge solution should correspond to a physical vacuum of the field theory. In agreement with expectations it is the flow that was claimed to describe the singular point on the moduli space.

We can also check the solution has the appropriate dimension moduli space and running coupling by brane probing. To do so we again rely on Pilch and Warner's ability to lift the $5 \mathrm{~d}$ solutions. They provide us with

$$
\begin{gathered}
d s^{2}=\Omega^{2}\left(e^{2 A} d x^{2}+d r^{2}\right)+\frac{\Omega^{2}}{\rho^{2}}\left(\frac{d \theta^{2}}{c}+\rho^{6} \cos ^{2} \theta\left(\frac{\sigma_{3}^{2}}{c X_{2}}+\frac{\sigma_{1}^{2}+\sigma_{2}^{2}}{X_{1}}\right)+\frac{\sin ^{2} \theta}{X_{2}} d \phi^{2}\right) \\
\Omega^{2}=\left(c X_{1} X_{2}\right)^{1 / 4} / \rho, \quad c=\cosh m \\
X_{1}=\cos ^{2} \theta+\rho^{6} c \sin ^{2} \theta, \quad X_{2}=c \cos ^{2} \theta+\rho^{6} \sin ^{2} \theta \\
C^{4}=\frac{e^{4 A} X_{1}}{\rho^{2}} d x^{0} \wedge d x^{1} \wedge d x^{2} \wedge d x^{3}
\end{gathered}
$$

Substituting into the Born Infeld action for a static probe D3 brand 10.12 we find the potential only vanishes in the $r-\phi$ plane at $\theta=\pi / 2$. The moduli space is indeed two dimensional. Allowing motion in the radial direction in this plane we can find a radial coordinate $u$ in which the kinetic term is canconical

$$
L \sim N \frac{\cosh m}{u^{2} \sinh m}\left(\dot{u}^{2}+u^{2} \dot{\phi}^{2}\right)
$$

In these coordinates the $m$ behaviour decouples from that of $\rho$. There are therefore a set of solutions, depending on the boundary condition on $\rho$, that have $\rho$ diverge at different values of $u$. We should cut off the flow of $m$ at this $u$ value since the SUGRA diverges. Inserting the behaviour of $m$ as a function of radial coordinate in the two asymptotic regimes we find that in the UV we return to the $\mathrm{N}=4$ value of the coupling and in the IR we find the form $N \log u / u_{0}$ where $u_{0}$ corresponds to the boundary condition on $m$. The coupling therefore correctly interpolates between the $\mathrm{N}=4$ beta function and the $\mathrm{N}=2$ IR beta function. It blows up at $u_{0}$. The flows with different $\rho\left(y_{U V}\right)$ describe different points on moduli space and the low energy coupling is this function evaluated at the value of $u$ where

rome00: submitted to World Scientific on November 16, 2018 
$\rho$ diverges corresponding to evaluating the beta function at the vev. Only for the critical flow discussed above does the divergence in $\rho$ fall within $u_{0}$ and here we must excise the solution at up because the probe's tension has fallen to zero - this is the enhancon mechanism of 13 . It is indicative that in the field theory new degrees of freedom become massless at these points on moduli space. In 12 the authors point out that to match the scalar kinetic term with the $F^{2}$ term's coupling one must also make an angular change of variables that squashes the enhancon ring at $u=u_{0}$ to a line segment.

\section{Summary}

We have seen therefore that the $A d S / C F T$ continues to provide a good description of the gauge theory physics when the $\mathrm{N}=4 \mathrm{SYM}$ is deformed with relevant operators. This suggests that many other interesting aspects of strong interation gauge dynamics may be open to a gravity description. One might even hope to eventually realize a gravity description of QCD. In the deformed theories one always suffers from an inability to decouple the $\mathrm{N}=4$ matter, since the $\mathrm{N}=4$ theory is strongly coupled in the UV, but these ideas will hopefully lead to a wider class of correspondences. Very recently this hope has begun to be realized as a number of pure $N=1$ and $\mathrm{N}=2$ theories have been shown to have gravity duals 14 .

\section{References}

1. J. Maldacena, Adv. Theor. Math. Phys. 2 (1998) 231, hep-th/9711200.

2. O. Aharony, S. Gubser, J. Maldacena, H. Ooguri , Y. Oz, Phys. Rept. 323 (2000) 183, hep-th/9905111.

3. E. Witten, Adv. Theor. Math. Phys. 2 (1998) 253, hep-th/9802150.

4. L. Girardello, M. Petrini, M. Porrati and A. Zaffaroni, JHEP 9812 (1998) 022, hep-th/9810126; J. Distler and F. Zamora, Adv. Theor. Math. Phys. 2 (1998) 1405, hep-th/9810206.

5. D. Z. Freedman, S. S. Gubser, K. Pilch and N. P. Warner, JHEP 0007 (2000) 038, hep-th/9906194

6. S.S. Gubser, hep-th/0002160; A. Brandhuber and K. Sfetsos, Phys. Lett. B488 (2000) 373, hep-th/0004148

7. K. Pilch and N. P. Warner, hep-th/0004063.

8. N. Evans, M. Petrini, Nucl. Phys. B592 (2000) 129-142, hep-th/0006048

9. M. Günaydin, L.J. Romans and N.P. Warner, Nucl. Phys. B272 (1986) 598.

10. N. Evans, C.V. Johnson, M. Petrini, JHEP 0010 (2000) 022, hep-th/0008081.

11. N. Dorey JHEP 9907 (1999) 021, hep-th/9906011; N. Dorey and S. Prem Kumar, JHEP 0002 (2000) 006, hep-th/0001103

12. A. Buchel, A.W. Peet, J. Polchinski, hep-th/0008076

13. C. V. Johnson, A. W. Peet and J. Polchinski, Phys. Rev. D61 (2000) 086001, hep-th/9911161.

14. J. Maldecena and C. Nunez, hep-th/0007018; I.R. Klebanov, M.J. Strassler, JHEP 0008:052,2000, hep-th/0007191; M. Bertolini, P. Di Vecchia, M. Frau, A. Lerda,R. Marotta and I. Pesando, hep-th/0011077; A. Rajaraman, hepth/0011279.

rome00: submitted to World Scientific on November 16, 2018 